\title{
FRAGMENTATION OF ASTEROIDS AND DELIVERY OF FRAGMENTS TO EARTH
}

\author{
G. W. WETHERILL
}

\begin{abstract}
Earth-impacting meteoroids are derived from both comets and asteroids, and some uncertainty still exists regarding with which of these bodies some stone meteorites should be identified. In contrast, the long cosmic ray exposure ages of iron meteorites strongly suggest a long-lived astercidal scurce capable of providing $\sim 10^{8} \mathrm{~g} / \mathrm{yr}$ of this material to the earth's surface over at least much of solar system history. Spectrophotometric data show that differentiated asteroids are concentrated in the inner portion of the asteroid belt. The orbital histories of fragments of inner belt asteroids are investigated, considering the combined effects of close planetary encounters, secular perturbations, and secular resonances. Particular attention is given to the low inclina. tion $\left(\leqslant 15^{\circ}\right.$ ) objects with small semimajor axis (2.1 to $\left.2.6 \mathrm{~A} . \mathrm{U}.\right)$, which can make fairly close approaches to Mars $(\leqslant 0.1$ A.U.). It is found that the annual yield and dynamical lifetime of collision fragments of these asteroids is in agreement with the observed impact rate and exposure age of iron meteorites. A smaller yield of stone meteorites $\left(\sim 10^{7} \mathrm{~g} / \mathrm{yr}\right)$ is expected, because elimination of these objects by collision is probable on the long dynamical time scale. Achondrites could be produced in this way; the yield is probably too low to account for chondrites. Chondrites could possibly be derived indirectly from these bodies insofar as these asteroids are also sources of Apollo and Amor objects.
\end{abstract}

Understanding the history of the solar systen requires the discovery of the qualitative principles which govern its long-term dynamical evolution. Thereby one may rise above the severo limitations imposed by mere numerical extrapolation of present orbits into the past. Furthermore, it is also necessary that these principles be at least approximately quantified to permit comparison of theory with observation. Our understanding of these principles began with the work of Copernicus and Kepler who showed that the heliocentric trajectories of bodies in the solar system can be approximated as ellipses, the parameters and orientation of which change only slowly with time. Further developments include the demonstration by Jacobi of the approximate constancy of the param . eter in the 3-body problem which bears his name. More recent1y, Opik (1951) emphasized the dominant effect of close encounter in the long-term evolution of planet-crossing bodies. Even though such close encounters are rare enough to be neglected on the $\sim 10^{4}$ year time scale imposed by practical limitations of numerical integration, on the real $\sim 10^{9}$ year time scale of the solar system, rather than being improbable or ad hoc, these encounters are inevitable. Arnold (1964, 1965) extended Bpik's work by development of a stochastic simulation of the 
solar system, the evolution of which was determined by close encouters as described by Opik. This work has been subsequently further elaborated (Wetherill and Williams 1968, Wetherill 1968a,b, 1969, 1971, 1975). Of similar importance has been the development of general theories of the long range planetary perturbations, which, subject to specific assumptions, are valid for all time (Brouwer 1951; Williams 1969).

Discussion of pussible sources of metcorites and meteoroids in the solar system requires development of these principles. Cosmic ray exposure ages show that the time interval between fragmentation of the source and impact of the fragment with the earth is typically millions of years and, in the case of iron meteorites, may be more than $1000 \mathrm{~m} . \mathrm{y}$. The general problem has been reviewed (Wetherill 1974) and the reader may refer to this earlier article for a more complete discussion with references. In this review it was concluded that major quantities of non-volatile fragments $\geqslant 1 \mathrm{~kg}$ in mass from both comets and asteroids impact the earth. Any of this material which survives passage through the atmosphere will be a meteorite. Although much conjecture is possible, understanding of the details of the internal structure of either comets or asteroids is too poor to permit any certainty in statements regarding so subtle a property as the mechanical strength of material in their interior. A difficult task therefore remains, that of identifying the various classes of meteorites with the various classes of comets and asteroids.

Derivation of massive meteoroids from comets presents no serious dynamical problem, as comets are observed to be in earth-crossing orbits, and non-volatile riateial ejected from comots or remaining after loss of volatile ices will remain for a time in very similar orbits. This is not the case for main belt asteroids, as velocity changes of $\sim 6 \mathrm{~km} / \mathrm{sec}$ are necessary to effect earth.crossing. Any plausible cquilibration of this kinetic energy of $2 \times 10^{11}$ ergs/g will result in fragmentation, melting, and othor shock effects far beyond those observed in meteorites. Therefore derjvation of metcorites, from asteroids has required understanding the mechanisms whereby asteroidal fragments can be gently transferred into earth-crossing orbits. These mechanisms are necessarily inefficient, as the annual flux of meteorites is only $\sim 10^{-5}$ of the meteorite-sized fragmentation debris produced in the asteroid belt per year. Anders (1964) and Arnold (1964, 1965) discussed the role of gravitation perturbaticns by Mars of mars-crossing fragments. Further work on this mechanism is the principal subject of the present article.

It has also been shown (Zimmerman and Wetheril1 1973) that a series of probable events involving resonant acceleration and libration in the $2: 1 \mathrm{Kirk}-$ wood gap can also gravitationally deflect a significant quantity $\left(\sim 10^{8} \mathrm{~g} / \mathrm{yr}\right)$ of asteroidal fragments to earth-crossing, although the quantitative yield from this source is difficult to calculate with any high degree of certainty. This mechanism has the property that the resulting orbits first become earth-crossing with perihelion very near $1 \mathrm{~A} . \mathrm{U}$, and aphelion beyond $4 \mathrm{~A} . \mathrm{U}$. This type of initial earth-crossing orbit has previously been shown to be the only kind which will yield the observed distribution of time of fall and radiant chondritic meteorites (Wetherill 1968b, 1971), and which will lead to the observed orbital distribution of Prairie Network firebal1s. At this meeting Ceplecha (1976) presentcd evidence tentatively associating about $1 / 3$ of these firebal1s (type I) with ordinary chondrites ard another $1 / 3$ (type II) with carbonaceous chondrites on the basis of their ablation and fragmentation in the atmosphere (Ceplecha and McCrosky 1976). Although their orbital distributions overlap considerably, probably significant differences are found in the distrihttion of semi-major axes of these two types of fireballs. The discovery fy Scholl and Froeschle (1976) that resonant acceleration in the $5: 2$ Kirkwood gap may be even more important than that in the $2: 1$ gap naturally gives rise to the speculation that Ceplecha's two fireball classes may be related to these two Kirkwood gap sources. However, 
much detailed work is required before this possibility should be taken very seriously.

There remain the Apol10 and Amor objects, earth-crossing of which must contribute to the terrestrial meteoroid flux. The role of these bodies as terrestrial meteoroid sources has recently been discussed (Wetherill 1976) and was also a principal subject of two papers (Simonenko 1976; Levin 1976) presented at this meeting. In many ways these objects, themselves of uncertain origin are attractive chondrite sources. However, serious problems with the predicted orbital distributions of their fragments remain to be resolved, hopefully not in an ad hoc way, before these bodies can be regarded as the principal sources of chondritic meteorites.

In this paper attention is directed to a particular group of meteorites the irons, the long exposure ages of which strongly suggest an asteroidal origin, inasmuch as any other plausible solar system source would impose a shorter ( $\leqslant 10^{8}$ year) dynamical 1 ifetime on the fragment.

In this discussion the effects of close planetary encounters and the longterm "secular" perturbations will be combined to investigate the orbital evolution of collision fragments derived from asteroidal bodies with semi-major axes in the range 2.1 to $2.6 \mathrm{~A} . \mathrm{ll}$. This problem has been previously studied by Arnold (1965) who assumed that only planetary encounters wore important. The subsequent discovery by williams (1969) of strong secular resonances in this region showed that neglect of secular perturbations is likely to lead to an unrealistic solar system model and possibly to incorrect conclusicns. It will be seen from the following discussion that although significant resemblance between these results and those of Arnold remain, there are important differences as well.

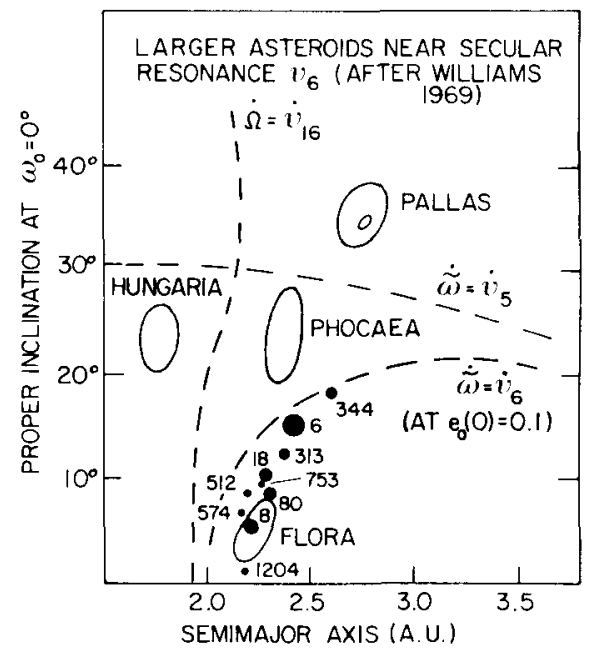

Figure 1. Inner region of the asteroid belt showing the secular resonances and the asteroids most productive of earth-crossing fragments.

The portion of the asteroid belt most demanding consideration is the densely population region below the resonant surface $\dot{\vec{u}}=\dot{v}$ (Figure 1 ), where large asteroids such as 4 Vesta, 6 Hebe, 8 Flora, 18 Melpomene, and 313 Chaldaea are found, as well as the expected accompanying mass spectrum of smaller bodies. Abovc this resonant surface are located two regions in which high inclination objects are found: the Phocaea and Hungaria regions. The largest 
body in the Hungaria region, 434 Hungaria, is only about $8 \mathrm{~km}$ in radius, which severely limits the importance of these bodies as major meteorite sources. Larger bodies are found in the Phocaea region, fragments of which could become Mars-crossers. The high inclination of these bodies and the large ejection veloicty required to place them in the resonant region suggest that these fragments would be too long-1ived to represent a major meteorite class. Although these asteroids merit further investigation, attention in this paper will be directed to the region below the resonant surface.

Most of these low inclination bodies are also of sufficiently low eccentricity to preclude close encounters with Mars, and as far as in known are entirely stable on the $4.5 \times 10^{9}$ year time scale of the solar system. A few objects, e.g., 512 Taurinensis are in orbits for which Mars encounters are

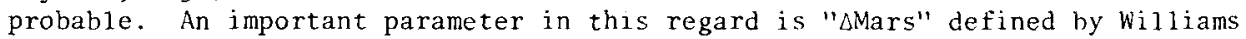
(1971) as the minimum possible distance of approach of the body to the orbit of Mars, assuming the most favorable possible combination of orbital elements of both the body and of Mars. Close encounters are possible only for those objects for which $\Delta$ Mars is negative. This is the casc for some of the objects in this region, e.g., 313 Chaldaea for which $\Delta$ Mars $=-.039$ A.U. However even though close encounters with Mars are in principle possible for this body, it can be showr that the precisely required combination of secular perturbation phases occur so infrequently that for practical purposes the orbit can be considered almost as stable as, for example, 8 Flora for which $\Delta$ Mars $=+.024$ A.U.

This stability does not necessarily apply to the orbits of fragments ejected following collisions between these bodies. For some of these asteroids, ejection at velocities of $\sim 300 \mathrm{~m} / \mathrm{sec}$ can place fragments into orbits for which $\Delta$ Mars is sufficiently negative (i.e. $\leqslant-.05$ A.U.) to lead to rapid instability. This can arise as a consequence of changes in semi-major axes (a) and eccentricity (e) resulting directly from the collision. More important, proximity to the resonant surface $\dot{\bar{\omega}}=\dot{v}_{6}$ will cause changes in (a) (and to lesser extent in inclination) to lead to large increases in (e) and hence to negative values of $\Delta$ Mars.

This effect has been quantitatively evaluated by calculation of the orbits of random ejecta from all known bodies in the region under consideration for which $\Delta$ Mars <+.10 A.U. as given by Williams (private communication 1975), as well as several other bodies particularly close to the resonant surface $\dot{\vec{u}}=\dot{v}_{6}$. It is assumed that ejection is isotropic in the reference frame of the target body and takes place at random positions in the orbit of this body, and at random values of the phases of the forced oscillations of secular perturbation theory (Brouwer and Van Woerkom 1950; Williams 1969). The mass yield from collisions is estimated by combining asteroid collision dynamics (Wetherill 1967) with the results of cratering studies (Gault et al. 1963, O'Keefe and Ahrens 1976); these cratering studies are also used to obtain the velocity distribution of the ejected fragments. When available, radii based on measured albedos (Morrison 1974, Morrison and Chapman 1976) were used in calculating yields. Otherwise it was assumed that the objects were "S" type asteroids (Chapman 1976) and the expression:

$$
\log r=3.30-0.2 \mathrm{~g}_{B}
$$

was used to calculate $r$, the radius in kilometers, from the absolute blue magnitude, $g_{B}$. When appropriate, this distribution is modified by the effect of the non-zero escape velocity of the target asteroid. The effect of the $\dot{v}_{6}$ resonance is included by use of the expressions

$$
\begin{aligned}
& e_{0} \sin \tilde{\omega}_{0}=e \sin \tilde{\omega}-M_{6} \sin v_{6} \\
& e_{0} \cos \tilde{\omega}_{0}=e \cos \tilde{\omega}-M_{6} \cos v_{6} .
\end{aligned}
$$


In these expressions e and $\bar{\omega}$ are defined by the orbit of the target asteroid and the velocity vector of cjection, $v_{6}$ by the randomly selected phase of forced oscillation. The forced oscillation amplitude in the vicinity of the resonance, $M_{6}$ is cbtained from calculations of Williams (private communication 1976). These equations can then be solved for the proper eccentricity $c_{0}$, which then permits calculation of the maximum eccentricity of the fragment $\left(e_{0}+\left|\Delta M_{6}\right|\right)$ and the new value of $\Delta$ Mars.

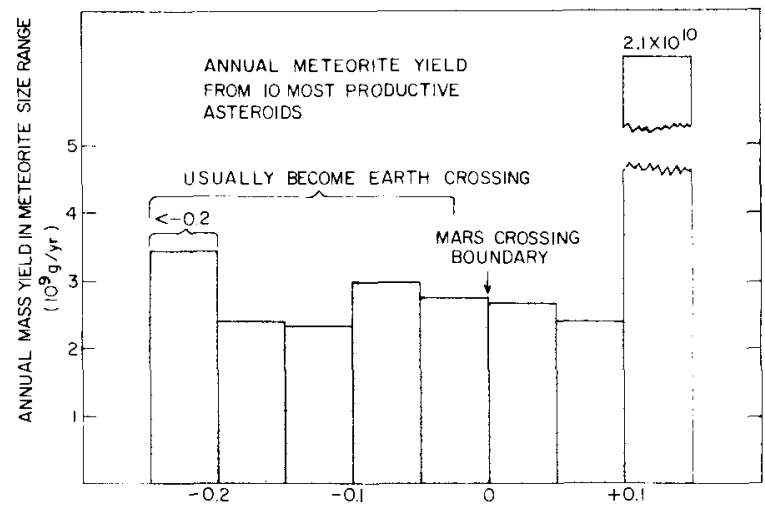

MINIMUM POSSIBLE DISTANCE FROM MARS (A.U.)

Figure 2. Calculated annual yield of meteoritic fragments from the asteroids shown in Figure 1 , as a function of the minimum possible encounter distance from Mars (MMars).

The results of these calculations are shown in Figure 2 . It is found that the yield of fragments with negative $\Delta$ Mars is dominated by a few bodies (those marked on Figure 1), and that the total yield of such fragments in the meteorite size range $\left(100 \mathrm{~g}\right.$ to $\left.10^{6} \mathrm{~g}\right)$ is $-10^{10} \mathrm{~g} / \mathrm{yr}$. The principal uncertainty in this calculation probably arises from uncertainties in the cratering data employed. Although no essential difference is found between use of the experimental data of Gault et al. (1963) and the theoretical results of O'Keefe and Ahrens (1976) this agreement could well be fortuitous. Inclusion of the resonance increases the yield by a factor of about 20 over that calculated otherwise.

The subsequent evolution of the orbits of these fragments was followed by use of the stochastic "Monte Carlo" approach previously employed, now modified to include new calculation of $e_{0}$ and $M_{6}$ by use of equations (2) following each planetary encounter. The ease with which Mars can perturb the semi-major axis of the fragment into the vicinity of the $v_{6}$ resonance and the consequent increasc in its maximum eccentricity results in earth-crossing frequently taking place within 100 million years (Figure 3). Earlier calculations, in which the effects of the resonarce were not included, gave the result that a 500 to 1000 m. $y$. interval was required for the random-walk reorientation of the crbit into an earth-crossing configuration.

Nevertheless this rapid achievement of earth-crossing does not decrease the time scale for earth impacts to the extent that might be expected. Earthcrossing occurs only at the maxima of the large amplitude forced oscillations in eccentricity; most of the time the object is only a Mars-crosser. In addition to the decrease in earth-impact probability resulting from earth-crossing occurring but a fraction of the time, it also turns out the continual Mars. crossing frequently permits Mars to perturb the semi-major axis away from the resonance and at least temporarily terminate earth-crossing. Quasipermanent earth-crossing and a high probability of earth-impact requires a 


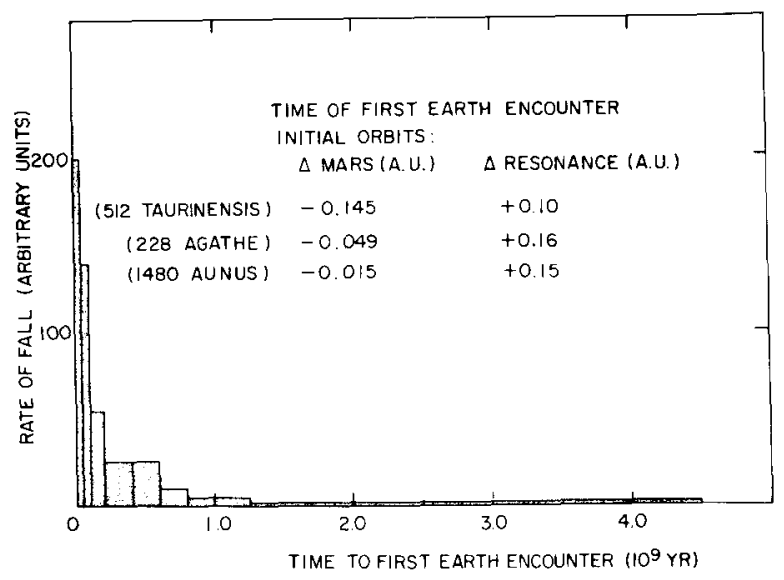

Figure 3. Time interval required for the first close encounter with earth for fragments ejected into typical orbits with values of sMars in the range indicated in Figure 1 as usually becoming earth-crossing.

"run" of earth-perturbations whercin the sign of the change in semi-major axis is predominantly positive or negative, removing the object from the influence of the resonance and "freezing in" the large ( 0.6$)$ eccentricity characteristic of initiai earth-crossing orbits. The time required for the occurrence of such runs may be long, $\sim 0.5 \times 10^{9}$ years. Calculations of the times for earth impact for objects with initial negative values of $\Delta$ Mars are given in Figure 4 . Al.. though the number of Monte Carlo runs calculated was not sufficient to provide high-quality statistics, the general result is clear: earth impact is most likely to occur between $100 \mathrm{~m} . \mathrm{y}$. and $1000 \mathrm{~m} . \mathrm{y}$. after the asteroidal collision which produced the fragment.

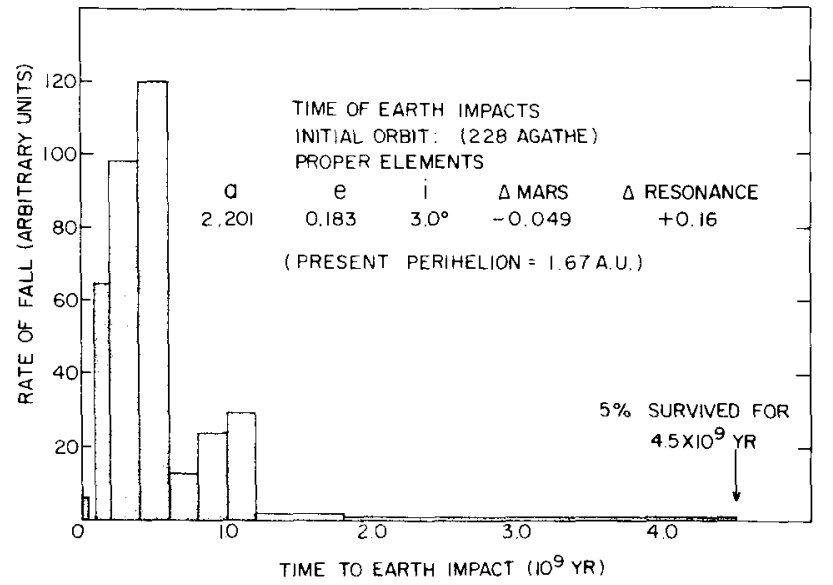

Eigure 4. Time intervals required for earth impact for a typical starting orbit.

This time scale is that actually found for iron meteorites, as indicated by their exposure ages (Voshage 1967). However a component with the short $(<100$ m.y.) exposure ages found for some irons is also compatible with this 
source, as a consequence of the rapidity with which initial earth-crossing can occur. These same Monte-Carlo calculations give an earth-impact efficienty of 5 to 10 percent. If it is assumed that $10 \%$ of the initial fragments are iron objects, this leads to a terrestrial impact rate of $\sim 10^{8} \mathrm{~g} / \mathrm{yr}$, in agreement with observation.

of course, it is not known whether or not the asteroids under consideration contain the necessary quantity of massive metalic iron. Spectrophotometric data suggests that differentiated bodies are predominantly located in this inner region of the asteroid be1t. Iron meteorites must come from somewhere, and if the plausible assumption is made that they come from asteroids there doesn't seem to be a better place in the asteroid belt to seck their source.

The long time required for earth impact greatly reduces the earth impact yicld expected for the silicate fragments produced in these asteroidal collisions, as a consequence of the vulnerability of the rock fragments to further collisional destruction. A yield of $\sim 10^{7} \mathrm{~g} / \mathrm{yr}$ of fragments on the $\leqslant 50 \times 10^{7}$ year time scale corresponding to stone meteorite exposure ages might be obtained. This could represent an important supply of achondrites but is probably inadequate as a source of chondrites, sources of which may be sought elsewhere (e.g., Wetherill 1976, Zimmerman and Wetheril1 1973). The distribution of radiants and time of fall expected for thesc orbits will probably turn out to be quite different from those found for chondritic meteorites (Wetherill 1968b); the smaller quantity of data for differentiated meteorites can be more casily reconciled with a wide range of predictions.

This discussion has been cast in the framework of the problem of identification of meteorite sources. The combined effect of the $v_{6}$ resonance and Mars perturbations has also been considered in the contexts of impact cratering on Mars and of the production rates and orbits of the small Amor and Apollo objects, from which chondrites may be derived (Levin et al. 1976, Wetherill 1976). These results will be presented clsewhere at a later time.

\section{REFERENCES}

Anders, E. 1964, Space Science Rev., 3, 583.

Arnold, J. R. 1964, in Isotopic and Cosmic Chemistry, 347, North Holland. Arnold, J. R. 1965, Astrophys. J., 141, 1536.

Brouwer, D. 1951, Astron. J., 56, 9.

Brouwer, D., and VanWoerkom, A.J.J. 1950, Astron. Papers L.S. Nava1 Obs. Naut. Almanac office 13 , Part $I I, 85$.

Ceplecha, Z. 1977, this volume.

Ceplecha, Z., and McCrosky, P. E. 1976, Center for Astrophysics, Preprint Series, No. 442 .

Chapman, C. R. 1976, Geochim. Cosmochim. Acta, 40, 701.

Gault, D. E., Shoemaker, E. M., and Moore, H. J. 1963, NASA TN 1767.

Levin, B. J. 1977, this volume.

Levin, B. J., Simonenko, A. N., and Anders, E. 1976, Icarus, in press.

Morrison, D. 1974, Astrophysics. J., 194, 203.

Morrison, D., and Chapman, C. R. 1976, Astrophys. J., 204, 934.

0'Keefe, J. D., and Ahrcns, T. J. 1976, Proc. 7th Lunar Sci. Conf., Pergamon, in press.

Opik, E. J. 1951, Proc. Roy. Irish Acad., 54A, 165.

Scholl, J., and Froeschlé, C. 1976, this volume.

Simonenko, A. N. 1977, this volume.

Voshage, H. 1967, 2. Naturforsch, 22a, 477.

Wetheri1l, G. W. 1967, J. Geophys. Res., 72, 2429. 


\section{WETHERILL}

Wetheri11, G. W. 1968a, in Origin and Distribution of the Elements, L. H. Ahrens, ed., pp. 423-443, Pergamon, Oxford.

Wetheri11, G. W. 1968b, Science, 159, 79.

Wetheri11, G. W. 1969, in Meteorite Research, P. Millman, ed. pp. 573-589, Reide1, Dordrecht.

Wetherill, G. W. 1971, in Physical Studies of the Minor Planets, T. Gehrols, ed. pp. 447-460, NASA SP. 267.

Wetherill, G. W. 1974, Ann. Rev. Earth Planet. Sci., 2, 303.

Wetherill, G. W. 1975, Proc. 6th Lunar sci. Conf, Pergamon, 1539.

Wetheri11, G. W. 1976, Geochim. Cosmochim. Acta, in press.

Wetheril1, G. W., and Williams, J. G. 1968, J. Geophys. Res., 73, 635.

Williams, J. G. 1969, Ph.D. dissertation, UCLA, 273 pp.

Williams. J. G. 1971, in Physical Studies of Minor Planets, T. Gehrels, Ed. pp. 177-181, NASA SP-267.

Zimmerman, P. D., and Wetheri11, G. W. 1973, Science, 182, 51.

\section{DISCUSSION}

EILD: If a group of meteorites originates in the asteroid belt, would all group members have been transferred to earth crossing orbit at once, or would they make the transfer one at a time?

WETHERILL: A group originating from a single collision would be transferred individually, one at a time, into earth-crossing. Some would never achieve earth-crossing before striking Mars or being ejected into interstellar orbits by Jupiter.

GROSSMAN: From your talk, I gather there is no easy way to get ordinary chondrites from the asteroid belt on the required short time-scale and, from other talks, I gather there are not many there anyway.

WETHERILL: If S-asteroids such as 6 Hebe and 8 Flora turn out to contain chondrites, in spite of their present identification as mesosiderites, then there may be a chance to derive ordinary chondrites from this portion of the asteroid belt, but I would have to press a number of parameters to the limit of uncertainty, and also might get into problems with simultaneously maintaining an adequate variety of iron meteorite parents. I think it most likely ordinary chondrites come from Apollo objects, but of course the source of Apollos is still poorly understood. The Kirkwood gap mechanism discussed by Zimmerman and myself could be important, as a meteorite source, but insofar as it is based on the $2: 1$ commensurability it seems more promising as a source of carbonacecus material if the spectrophotometric identifications are valid.

ANDERS: Do any of these objects have orbits resembling those of the Apollo or Amor asteroids?

WETHERILL: Yes, a large number of them do, but a high percentage evolve into very small orbits, frequently with aphelion near venus. No such objects are known but they would be harder to discover than Apollos or Amors, which are already difficult.

WASSON: It is important to bear in mind that there are a number of different chondrite groups, but about $90 \%$ of the mass flux consists of ordinary chondrites.

WETHERILL: Yes, and the problem is that if we accept the spectrophotometric 


\section{FRAGMENTATION OF ASTEROIDS}

meteorite class identifications, the mechanism discussed here does not provide an adequate mass yield of ordinary chondrites.

WASSON: Chondrite cosmic ray ages are all short ( $\$ 40 \mathrm{Myr}$ ), independent of groups. Since it seems reasonable that the parent bodies of these groups are at different solar system locations, it seems unlikely that the cosmic ray ages are of dynamic origin, and more likely that they are determined by erosional or fragmentation processes.

WETHERILL: I think it is open question whether the chondrite exposure ages are primarily controlled by collisions or by dynamics. The observed ages are very similar to the dynamic lifetime for a wide variety of earth-crossing orbits. Hence any mechanism which achieves earth-crossing on a shorter time scale will result in dynamic control. Apollo asteroids and cometary sources, Kirkwood gap resonances, and direct injection into $v_{6}$ or $v_{5}$ resonances have this property. The problem is to plausibly associate an adequate mass of ordinary chondrite parents with these mechanisms. The longer (up to $100 \mathrm{M.Y}$.) exposure ages of enstatite achondrites also suggest to me that the collisional lifetime of interplanetary stones is longer than the typical chondrite exposure age, but I would not insist on this. 